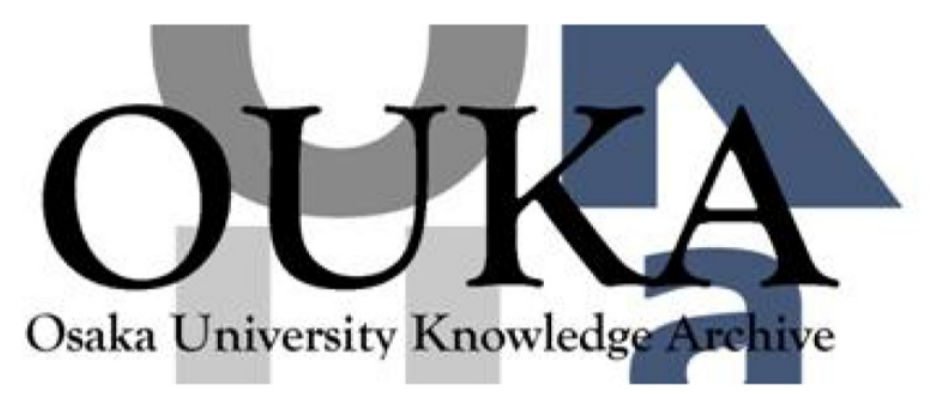

\begin{tabular}{|c|l|}
\hline Title & $\begin{array}{l}\text { A new facet of the reaction of nitro } \\
\text { heteroaromatic compounds with ethyl } \\
\text { isocyanoacetate }\end{array}$ \\
\hline Author(s) & $\begin{array}{l}\text { Murashima, Takashi; Fujita, Kenichi; Ono, Kazuo } \\
\text { et al. }\end{array}$ \\
\hline Citation & $\begin{array}{l}\text { JOURNAL OF THE CHEMICAL SOCIETY-PERKIN } \\
\text { TRANSACTIONS 1. 12 p. 1403-p. 1407 }\end{array}$ \\
\hline Issue Date & 1996-06-21 \\
\hline oaire:version & VoR \\
\hline URL & https://hdl. handle. net/11094/3215 \\
\hline rights & \\
\hline Note & \\
\hline
\end{tabular}

Osaka University Knowledge Archive : OUKA

https://ir. Library. osaka-u. ac. jp/

Osaka University 


\title{
A new facet of the reaction of nitro heteroaromatic compounds with ethyl isocyanoacetate
}

\author{
Takashi Murashima, ${ }^{*, a}$ Ken-ichi Fujita, ${ }^{a}$ Kazuo Ono, ${ }^{a}$ Takuji Ogawa, ${ }^{a}$ \\ Hidemitsu Uno ${ }^{b}$ and Noboru Ono ${ }^{*, a}$ \\ ${ }^{a}$ Department of Chemistry, Faculty of Science, Ehime University, Bunkyo-cho 2-5, \\ Matsuyama 790, Japan \\ ${ }^{b}$ Advanced Instrumentation Center for Chemical Analysis, Ehime University, Bunkyo-cho 2-5, \\ Matsuyama 790, Japan
}

\begin{abstract}
Nitro heteroarenes react with ethyl isocyanoacetate in the presence of 1,8-diazabicyclo[5.4.0]undecene (DBU) to give pyrroles or pyrimidine $N$-oxide depending on the structure of the starting nitro compounds. For example, 4-nitro-2,1,3-benzothiadiazole 3a reacted with ethyl isocyanoacetate to give ethyl 2,1,3-benzothiadiazolo[3,4-c]pyrrole-2-carboxylate $4 a(33 \%)$, while a similar reaction with 5 -nitro-2,1,3-benzothiadiazole $3 \mathrm{~b}$ gave the corresponding compound $4 \mathrm{~b}(21 \%)$ as a sole product. A plausible mechanism for these reactions is presented.
\end{abstract}

Since substituted pyrroles are of great interest in the preparation of functional compounds such as porphyrins and polypyrroles, many syntheses of them have been developed. ${ }^{1}$ Compounds containing a pyrrole ring such as the isoindoles are important for the preparation of conducting polypyrroles with low band-gaps and highly conjugated porphyrins, but there are no good general methods for obtaining them. ${ }^{2}$ Recent pyrrole syntheses from nitroalkenes and isocyanoacetates open a new way to the introduction of various substituents into the $\beta$-positions of the pyrrole ring. ${ }^{3,4}$ Furthermore, we and Lash have found that polycyclic aromatic nitro compounds react with isocyanoacetates in the presence of 1,8-diazabicyclo[5.4.0] undecene (DBU) to give the pyrroles fused with aromatic rings, and the pyrroles thus obtained are useful precursors for low band-gap polypyrroles ${ }^{3 a}$ or highly conjugated porphyrins. ${ }^{5}$ If this pyrrole synthesis can be extended to nitroalkenes substituted with heteroatoms, pyrroles substituted with heteroatoms can be prepared. However, the reaction of 2-nitro enamines or 2-nitroalkenyl sulfides with ethyl isocyanoacetate failed to give the expected pyrroles, 1hydroxypyrazoles being obtained instead. ${ }^{6}$ Thus, nitroalkenes having heteroatoms at the $\beta$-position react with isocyanoacetates in a different way from those of simple nitroalkenes. In this paper we deal with the reaction of nitro heteroaromatics with isocyanoacetates, in which a third aspect of the reaction of nitro compounds with isocyanoacetates is presented, namely the base-catalysed reaction of heteroaromatic nitro compounds with ethyl isocyanoacetate to give the corresponding pyrroles or pyrimidine $N$-oxides depending on the starting nitro compounds.

\section{Results and discussion}

During our study of the synthesis of the annulated pyrroles mentioned above, we found that 5-nitroquinoline 1a reacted with ethyl isocyanoacetate in the presence of DBU to give the pyrimidine $N$-oxide $2 \mathrm{a}(5 \%)$ instead of the corresponding and expected pyrroles. In this reaction, most of starting material was recovered unchanged from the reaction mixture. Some similar examples are listed in Table 1.

6-Nitroquinoxaline $\mathbf{1 b}$ and its derivatives $1 \mathbf{c}, \mathbf{d}$ gave the pyrimidine $N$-oxides $\mathbf{2 b}$-d whilst 2-nitrothiophene $1 \mathbf{e}$ under similar condition gave pyrimidine $N$-oxide $2 \mathrm{e}(40 \%)$. Compounds $2 a-e$ were identified from their NMR and high
Table 1 The fused pyrimidine $\mathrm{N}$-oxides $2 \mathbf{a}-\mathbf{f}$ were prepared in THF as solvent at room temperature; the reaction time was $48 \mathrm{~h}$ except for $2 \mathrm{f}$ for which it was $24 \mathrm{~h}$

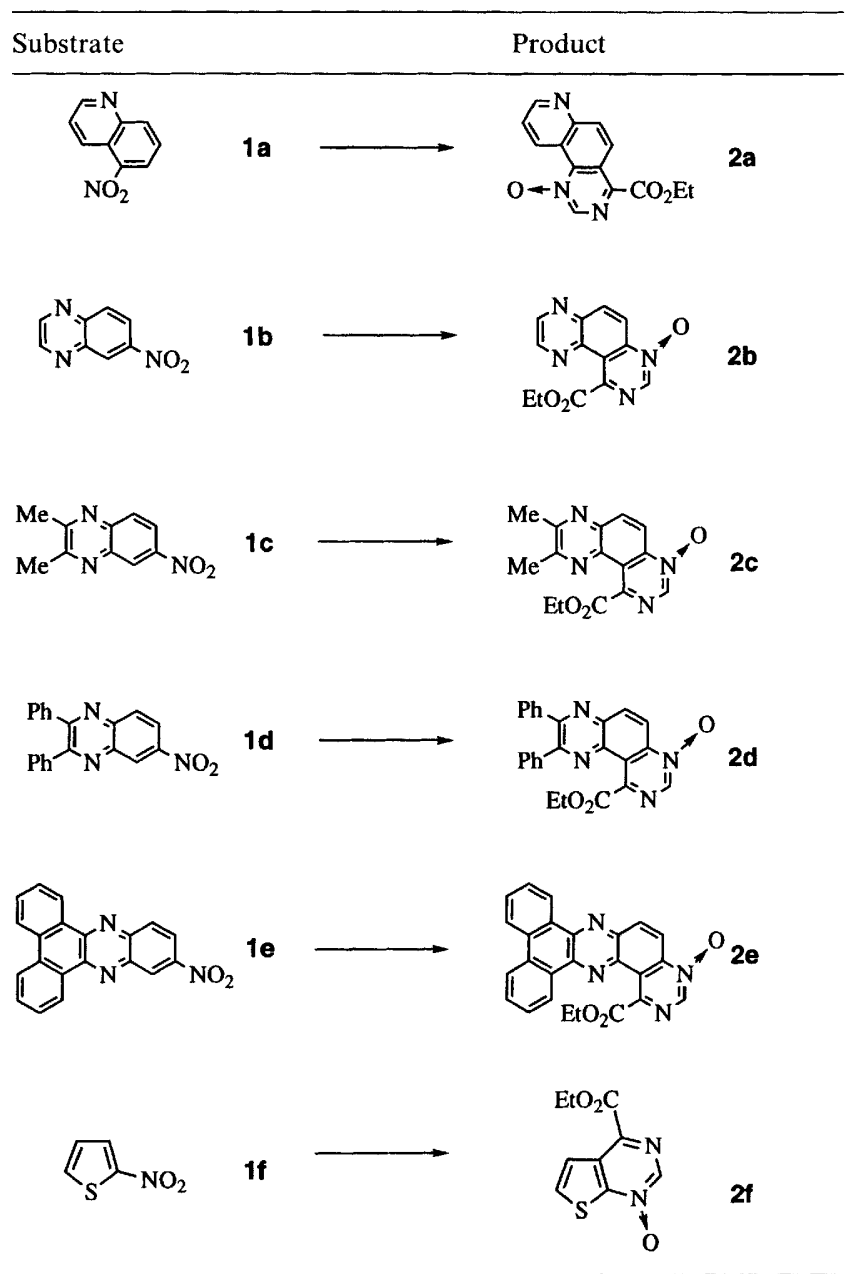

resolution mass spectral results; the position of the oxygen was confirmed for compound $2 \mathrm{e}$ by conversion of the latter into pyrimidine on treatment with $\mathrm{PCl}_{3}$.

Since pyrimidine bases exist in nature as nucleosides, 
the constituents of nucleic acid, they have attracted much attention. Although there are numerous methods for construction of the pyrimidine ring, ${ }^{7}$ this is not so for the direct synthesis of pyrimidine $\mathrm{N}$-oxides and the corresponding annulated compounds. An alternative method of preparing pyrimidine $\mathrm{N}$-oxides is by $\mathrm{N}$-oxidation of the corresponding pyrimidines with peracetic acid, although this gives both a mixture of 1 - and 3 -oxides ${ }^{8}$ and the possibility of side reactions. ${ }^{9}$

For these reasons, we decided to develop a convenient method of pyrimidine ring synthesis, although in this paper the main stress falls on the diversity of the reactions between aromatic nitro compounds and ethyl isocyanoacetate. From this point of view the reactions of the nitro-2,1,3-benzothiadiazoles $\mathbf{3 a}, \mathbf{b}$ were particularly noteworthy since the position of the nitro group was the sole factor determining the product. 4-Nitro-2,1,3-benzothiadiazole 3a reacted with ethyl isocyanoacetate in the presence of DBU to give the pyrrole $4 \mathbf{a}$ as the sole product. This reaction was conducted as follows. DBU (1 equiv.) was added dropwise to a stirred solution containing 1 equiv. each of 4-nitro-2,1,3-benzothaidiazole 3a and ethyl isocyanoacetate in dry THF kept at ca. $0^{\circ} \mathrm{C}$ in an ice bath. After the addition the mixture was allowed to rise slowly to ambient temperature. After $5 \mathrm{~h}$, the reaction was quenched with hydrochloric acid and the mixture worked up to give a brown solid, which was column chromatographed $\left(\mathrm{SiO}_{2}, \mathrm{CHCl}_{3}\right)$ to give the pyrrole 4a $(33 \%)$ In contrast, use of 5-nitro-2,1,3benzothiadiazole $\mathbf{3 b} \mathbf{b}^{10}$ as the starting material under similar reaction conditions gave the pyrimidine $\mathrm{N}$-oxide $\mathbf{4 b}$ as the sole product with recovery of most of the starting material. HPLC analysis and GC-MS measurement showed that there was no cross-contamination of the two products.

The homologues of 3a and 3b, the nitro-2,1,3-benzoselenadiazoles $5 \mathbf{a}, \mathbf{b}$ were prepared by a literature procedure (Scheme 1): ${ }^{10 b}$ thus, condensation of $o$-phenylenediamine with selenium dioxide followed by nitration with mixed acid gave 4-nitro2,1,3-benzoselenadiazole 5a whilst use of 4-nitrophenylene1,2-diamine in place of $o$-phenylenediamine gave 5-nitro-2,1,3benzoselenadiazole $\mathbf{5 b}$.

These selena compounds $5 \mathbf{a}$ and $\mathbf{5 b}$ with ethyl isocyanoacetate in the presence of DBU gave results similar to those of the nitro2,1,3-benzothiadiazoles 3a and 3b; see Scheme 1 and Table 2.

Proposed mechanisms for the formation of pyrroles and pyrimidines are illustrated in Scheme 2. Initial attack of the ethyl isocyanoacetate anion occurred at the $\beta$-position to the nitro groups to form the anionic intermediate 7 . When the nitro group was co-planar with the aromatic ring, this intermediate could be represented by two resonance structures 7 and 8 owing to the ambident character of the nitro group. Subsequent cyclization of the intermediate 7 gave the annulated pyrrole 9 whilst, for 8 , an intramolecular reaction of the carbon atom of the isocyanide moiety and the oxyanion yielded the annulated pyrimidine $N$-oxide $\mathbf{1 0}$.

In order to study these reactions, an equimolar mixture of $\mathbf{3 a}$ and 3b was allowed to react with an equivalent each of ethyl isocyanoacetate and DBU.

Since the pyrimidine $\mathrm{N}$-oxide $\mathbf{4 b}$ was formed in lower yield than the pyrrole 4a (see Table 2), we thought that the major product of this competitive reaction would be $4 a$. This, however, was not the case, the product formed being mainly the pyrimidine $\mathrm{N}$-oxide $\mathbf{4 b}$ derived from $\mathbf{3 b}$ together with a little 4a formed from 3a. These unexpected results suggest that the carbanion of ethyl isocyanoacetate attacks the 4-position of $\mathbf{3 b}$ more readily than the 5-position of $\mathbf{3 a}$ and that the Michael adduct $\mathbf{1 2}$ from $\mathbf{3 b}$ and ethyl isocyanoacetate carbanion is produced with greater facility than 11 (Scheme 3 ).

Further, in the case of the Michael adduct 11, subsequent cyclization occurs rapidly to give the corresponding pyrrole $\mathbf{4 a}$ whilst, in contrast, the stability of the Michael adduct 12 slows its cyclization. Such a hypothesis reasonably explains the yields

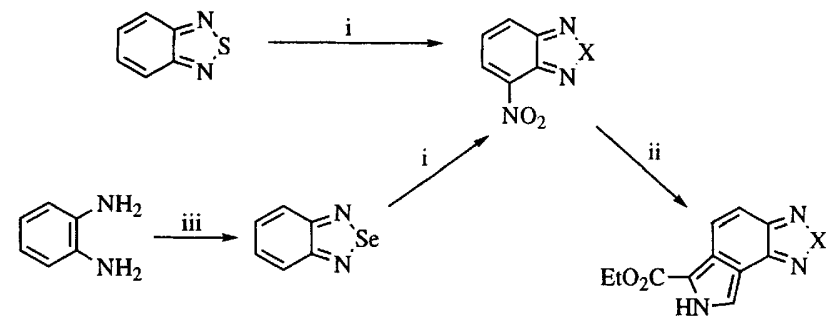

$$
\text { 岱 }
$$

Scheme 1 Reagents and conditions: i, $\mathrm{HNO}_{3}, \mathrm{H}_{2} \mathrm{SO}_{4}, 0^{\circ} \mathrm{C}, 5 \mathrm{~h}$; ii, $\mathrm{CNCH}_{2} \mathrm{CO}_{2} \mathrm{Et}$, DBU, THF, RT, 5 h; iii, $\mathrm{SeO}_{2}, \mathrm{EtOH}, \mathrm{AcOH}$, reflux, $2 \mathrm{~h}$; iv, $\mathrm{SOCl}_{2}, \mathrm{Et}_{3} \mathrm{~N}, \mathrm{RT}, 3 \mathrm{~h}$

Table 2 The nitrobenzothiadiazoles 3a,b and selenadiazoles $\mathbf{5 a , b}$ reacted with ethyl isocyanoacetate in THF at room temperature for $5 \mathrm{~h}$<smiles></smiles>

of $4 a$ and $4 b$ in the independent reaction of $3 a$ and $3 b$ with an equimolar proportion of ethyl isocyanoacetate.

Finally, the pyrimidine $N$-oxides $4 \mathbf{b}$ and $6 \mathbf{b}$ were produced not from the intermediate $\mathbf{1 2}$ but from the resonance structure 13, an intermediate capable of existence only when the nitro group is co-planar with the aromatic ring. Therefore, the pyrroles $4 \mathbf{a}$ and $6 \mathbf{a}$ were the sole products obtained from the highly hindered intermediate 11. The last assumption is supported by a PM3 calculation.

In summary, we have described the diversity of the reactions of aromatic nitro compounds with ethyl isocyanoacetate in a proposed mechanism, the detail of which and the validity of the assumption which underlie it are now under investigation.

\section{Experimental}

\section{General procedures}

Melting points were measured on a Yanaco hot-stage apparatus and are uncorrected. ${ }^{1} \mathrm{H}$ and ${ }^{13} \mathrm{C}$ NMR spectra were recorded on a JEOL-JNM-GSX 270 or JNM-EX 400 spectrometer at 


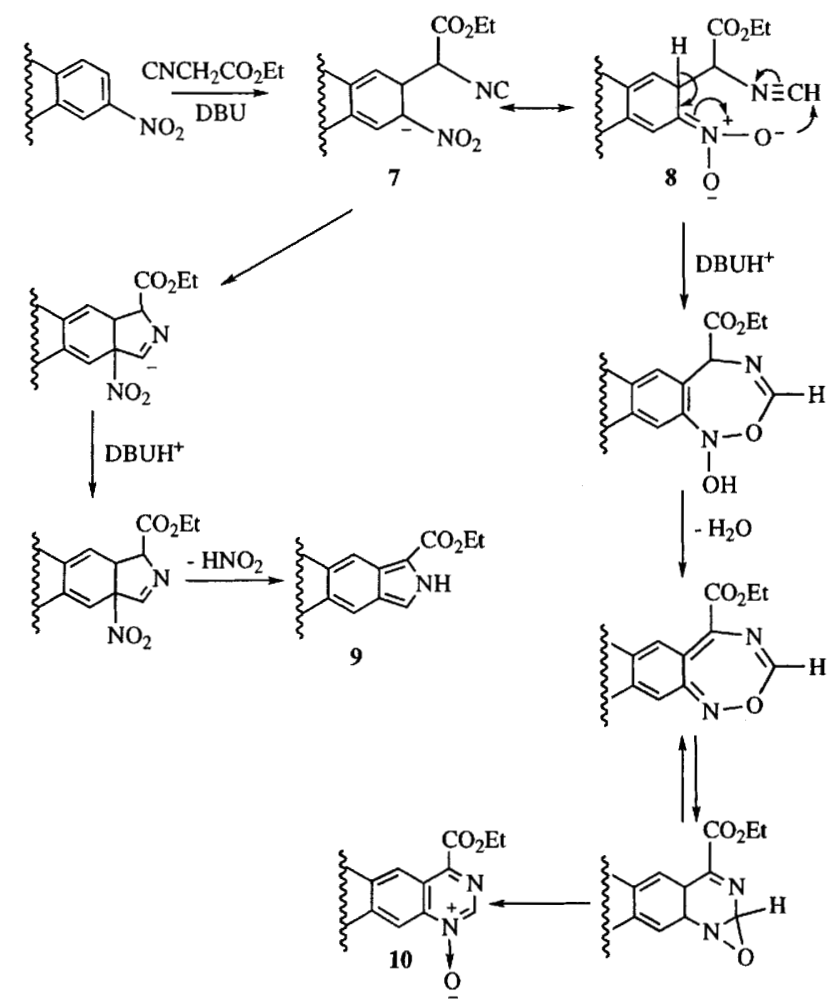

Scheme 2 Proposed mechanism for the formation of pyrrole and pyrimidine $N$-oxide
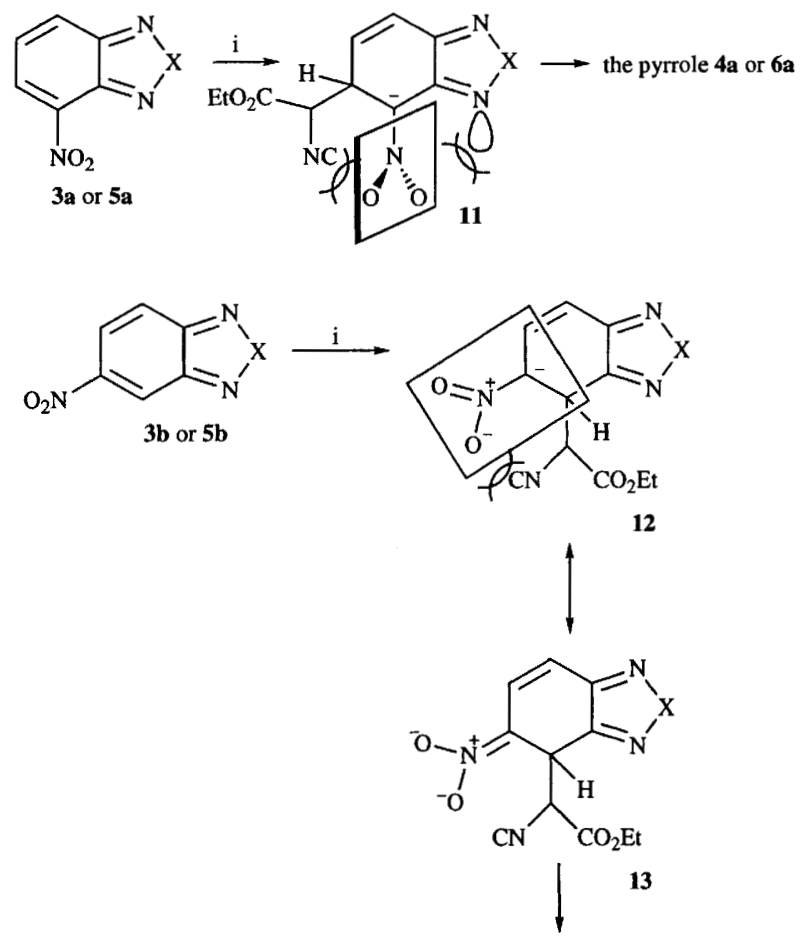

Pyrimidine $4 b$ or $6 b$

Scheme 3 Reagents and conditions: i, $\mathrm{CNCH}_{2} \mathrm{CO}_{2} \mathrm{Et}$, DBU, THF, RT, $5 \mathrm{~h}, \mathrm{X}=\mathrm{S}, \mathrm{Se}$

ambient temperature with $\mathrm{CDCl}_{3}$ or $\left[{ }^{2} \mathrm{H}_{6}\right]$ acetone as a solvent and tetramethylsilane as an internal standard; $J$ values are given in Hz. IR and UV-visible spectra were obtained with a Hitachi 270-30 and Shimadzu UV-2200 spectrophotometer, respectively. Mass spectra and high-resolution mass spectra were measured with a Hitachi M80B-LCAPI spectrometer under the following ionizing conditions: electron impact, $20 \mathrm{eV} ; 70 \mathrm{eV}$ for HRMS; high boiling PFK as a standard. THF was freshly distilled from sodium benzophenone ketyl. Ethyl isocyano- acetate was prepared from ethyl $N$-formylglycinate using $\mathrm{POCl}_{3}$ and triethylamine. ${ }^{11}$ Unless otherwise specified, all nitro aromatic compounds not commercially available were prepared by the conventional nitration of heteroaromatics using $\mathrm{HNO}_{3}-\mathrm{Ac}_{2} \mathrm{O}$ or $\mathrm{HNO}_{3}-\mathrm{H}_{2} \mathrm{SO}_{4}$.

\section{Ethyl pyrido[2,3- $h$ ]quinazoline-4-carboxylate 1-oxide 2a}

DBU $(1.67 \mathrm{~g}, 11 \mathrm{mmol})$ was added dropwise to a solution of 5nitroquinoline $1 \mathrm{a}(1.74 \mathrm{~g}, 10 \mathrm{mmol})$ and ethyl isocyanoacetate $(1.24 \mathrm{~g}, 11 \mathrm{mmol})$ in THF $\left(50 \mathrm{~cm}^{3}\right)$ at $0^{\circ} \mathrm{C}$. The resulting mixture was stirred at ambient temperature for $48 \mathrm{~h}$ after which it was treated with dilute hydrochloric acid $\left(50 \mathrm{~cm}^{3}\right)$ and extracted with ethyl acetate $\left(3 \times 20 \mathrm{~cm}^{3}\right)$. The combined extracts were washed with aq. sodium hydrogen carbonate, water and brine, dried $\left(\mathrm{Na}_{2} \mathrm{SO}_{4}\right)$ and evaporated. The residue was purified by silica gel column chromatography (ethyl acetate-hexane) to give the title compound $2 \mathrm{a}(0.132 \mathrm{~g}, 5 \%$ yield), mp $176-179^{\circ} \mathrm{C} ; \delta_{\mathrm{H}}\left(\mathrm{CDCl}_{3}\right) 1.57(\mathrm{t}, 3 \mathrm{H}, J 7.02), 4.66(\mathrm{q}$, $2 \mathrm{H}, J$ 7.18), 7.62 (d, $1 \mathrm{H}, J$ 8.24), 8.62-8.77 (m, $3 \mathrm{H}), 8.88-8.92$ $(\mathrm{m}, 1 \mathrm{H})$ and $9.55(\mathrm{~s}, 1 \mathrm{H}) ; \delta_{\mathrm{C}}\left(\mathrm{CDCl}_{3}\right) 13.71\left(\mathrm{CH}_{2} \mathrm{CH}_{3}\right), 62.59$ $\left(\mathrm{CH}_{2} \mathrm{CH}_{3}\right), 120.08,120.39,121.45,122.63,125.53,127.75$, $138.90,142.33,150.35,154.65,154.67$ and $163.58(\mathrm{C}=\mathrm{O})$; $v_{\max }(\mathrm{KBr}) / \mathrm{cm}^{-1} 3425,2930,2369,1718,1548,1375,1345,1293$, 1229,1204 and $1129 ; \lambda_{\max }\left(\mathrm{CH}_{2} \mathrm{Cl}_{2}\right) / \mathrm{nm} \mathrm{384,344.5,284.6}$ and 240 [Found (HRMS): $M, 269.0776$. Calc. for $\mathrm{C}_{14} \mathrm{H}_{11} \mathrm{~N}_{3} \mathrm{O}_{3}: M$, 269.0801].

The pyrimidine $N$-oxides $\mathbf{2 b}-\mathbf{f}$ were prepared by a similar procedures to that described for the preparation of $2 \mathbf{a}$, under the conditions described in Table 1.

Ethyl pyrazino[2,3-f]quinazoline-10-carboxylate 7-oxide $\mathbf{2 b}$. Yield $8 \% ; \mathrm{mp} 194-197^{\circ} \mathrm{C} ; \delta_{\mathrm{H}}\left(\mathrm{CDCl}_{3}\right) 1.48(\mathrm{t}, 3 \mathrm{H}, J 7.33), 4.64$ $(\mathrm{q}, 2 \mathrm{H}, J$ 7.17), 8.83-9.12(m, $4 \mathrm{H})$ and $9.36(\mathrm{~s}, 1 \mathrm{H}) ; \delta_{\mathrm{C}}\left(\mathrm{CDCl}_{3}\right)$ $13.96\left(\mathrm{CH}_{2} \mathrm{CH}_{3}\right), 62.66\left(\mathrm{CH}_{2} \mathrm{CH}_{3}\right), 135.57,135.67,137.41$, $142.00,142.75,144.32,144.63,144.71,146.64,147.21$ and $164.81(\mathrm{C}=\mathrm{O}) ; v_{\max }(\mathrm{KBr}) / \mathrm{cm}^{-1} 1742,1522,1260,1222,1198$,

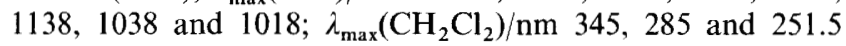
[Found (HRMS): M, 270.0756. Calc. for $\mathrm{C}_{13} \mathrm{H}_{10} \mathrm{~N}_{4} \mathrm{O}_{3}: M$, $270.0754]$.

Ethyl 2,3-dimethylpyrazino[2,3-f] quinazoline-10-carboxylate 7-oxide 2c. Yield $15 \%$; mp 184-187 ${ }^{\circ} \mathrm{C} ; \delta_{\mathrm{H}}\left(\mathrm{CDCl}_{3}\right) 1.46(\mathrm{t}, 3 \mathrm{H}, J$ $7.32), 2.81(\mathrm{~s}, 3 \mathrm{H}), 2.83(\mathrm{~s}, 3 \mathrm{H}), 4.63(\mathrm{q}, 2 \mathrm{H}, J 7.17), 8.41(\mathrm{~d}, 1$ $\mathrm{H}, J 9.46), 8.77(\mathrm{~d}, 1 \mathrm{H}, J 9.46)$ and $9.36(\mathrm{~s}, 1 \mathrm{H}) ; \delta_{\mathrm{C}}\left(\mathrm{CDCl}_{3}\right)$ $13.91\left(\mathrm{CH}_{2} \mathrm{CH}_{3}\right), 22.93(\mathrm{Me}), 61.45\left(\mathrm{CH}_{2} \mathrm{CH}_{3}\right), 135.75,136.22$, $140.64,144.35,144.49,144.78,154.21,156.50,161.36,165.92$ and $169.41(\mathrm{C}=\mathrm{O}) ; v_{\max }(\mathrm{KBr}) / \mathrm{cm}^{-1} 1744,1576,1446,1352$ and 1266; $\lambda_{\max }\left(\mathrm{CH}_{2} \mathrm{Cl}_{2}\right) / \mathrm{nm} \mathrm{345,285.5}$ and 251 [Found (HRMS): $\mathrm{M}, 298.1060$. Calc. for $\left.\mathrm{C}_{15} \mathrm{H}_{14} \mathrm{~N}_{4} \mathrm{O}_{3}: M, 298.1067\right]$.

Ethyl 2,3-diphenylpyrazino[2,3-f]quinazoline-10-carboxylate 7-oxide 2d. Yield $32 \%$; mp $184-187^{\circ} \mathrm{C} ; \delta_{\mathrm{H}}\left(\mathrm{CDCl}_{3}\right) 1.07(\mathrm{t}, 3 \mathrm{H}, J$ $7.02), 4.34$ (q, $2 \mathrm{H}, J 7.18), 7.35-7.47(\mathrm{~m} 5 \mathrm{H}), 7.55-7.60(\mathrm{~m}, 5$ $\mathrm{H}), 8.56(\mathrm{~d}, 1 \mathrm{H}, J 9.47), 8.88(\mathrm{~d}, 1 \mathrm{H}, J 9.46)$ and $9.34(\mathrm{~s}, 1 \mathrm{H})$; $\delta_{\mathrm{C}}\left(\mathrm{CDCl}_{3}\right) 13.56\left(\mathrm{CH}_{2} \mathrm{CH}_{3}\right), 62.46\left(\mathrm{CH}_{2} \mathrm{CH}_{3}\right), 120.19,120.29$, $128.21,128.47,129.69,129.83,130.26,136.16,136.39,137.66$, $137.86,140.93,142.68,143.79,144.70,145.06,153.65,155.59$ and $165.84(\mathrm{C}=\mathrm{O}) ; v_{\max }(\mathrm{KBr}) / \mathrm{cm}^{-1} 1744,1360,1274,1242$, 1186, 1140, 1024, 762 and $714 ; \lambda_{\max }\left(\mathrm{CH}_{2} \mathrm{Cl}_{2}\right) / \mathrm{nm} 413.5,394.5$, 328, 261.5 and 228.5; $m / z 422\left(\mathrm{M}^{+}\right)$(Found: $\mathrm{C}, 71.0 ; \mathrm{H}, 4.5 ; \mathrm{N}$, 13.15. $\mathrm{C}_{25} \mathrm{H}_{18} \mathrm{~N}_{4} \mathrm{O}_{3}$ requires $\mathrm{C}, 71.08 ; \mathrm{H}, 4.29 ; \mathrm{N}, 13.26 \%$ ).

Ethyl dibenzo[ $[a, c]$ pyrimido $[5,4-h]$ phenazine-15-carboxylate 12-oxide 2e. Yield $25^{\circ} ; \mathrm{mp} 292-295^{\circ} \mathrm{C} ; \delta_{\mathrm{H}}\left(\mathrm{CDCl}_{3}\right) 1.32(\mathrm{t}, 3 \mathrm{H}$, $J$ 7.32), 4.75 (q, $2 \mathrm{H}, J 7.17), 7.76-7.91(\mathrm{~m} 4 \mathrm{H}), 8.59-8.62(\mathrm{~m}, 3$ H), $8.87(\mathrm{~d}, 1 \mathrm{H}, J 9.76), 9.22(\mathrm{~d}, 1 \mathrm{H}, J 8.24), 9.34(\mathrm{~d}, 1 \mathrm{H}, J 7.93)$ and $9.38(\mathrm{~s}, 1 \mathrm{H}) ; v_{\max }(\mathrm{KBr}) / \mathrm{cm}^{-1} 1732,1272,1242,1194$ and

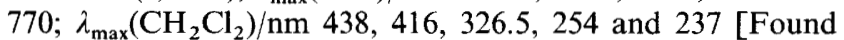
(HRMS): $\mathrm{M}, 420.1227$. Calc. for $\left.\mathrm{C}_{25} \mathrm{H}_{16} \mathrm{~N}_{4} \mathrm{O}_{3}: M, 420.1223\right]$.

Ethyl thieno[2,3- $d]$ pyrimidine-4-carboxylate 1-oxide 2f. Yield $40 \% ; \mathrm{mp} 173{ }^{\circ} \mathrm{C} ; \delta_{\mathrm{H}}\left(\mathrm{CDCl}_{3}\right) 1.51(\mathrm{t}, 3 \mathrm{H}, J 7.17), 4.75(\mathrm{q}, 2 \mathrm{H}, J$ $7.22), 7.77(\mathrm{~d}, 1 \mathrm{H}, J 5.80), 8.17(\mathrm{~d}, 1 \mathrm{H}, J 5.80)$ and $9.06(\mathrm{~s}, 1 \mathrm{H})$; $\lambda_{\max }\left(\mathrm{CH}_{2} \mathrm{Cl}_{2}\right) / \mathrm{nm} \mathrm{341,289}$ and $247 ; m / z 226\left[\mathrm{M}^{+}\left({ }^{34} \mathrm{~S}\right), 6 \%\right]$, $225\left[\mathrm{M}^{+}\left({ }^{33} \mathrm{~S}\right), 12\right], 224\left[\mathrm{M}^{+}\left({ }^{32} \mathrm{~S}\right), 100\right], 152(96)$ and $127(56)$. 
The structure of $2 \mathbf{f}$ was confirmed by treating the compound with $\mathrm{PCl}_{3}$ and converting it into the corresponding pyrimidine 2g; $\mathrm{mp} 79-80^{\circ} \mathrm{C} ; \delta_{\mathrm{H}}\left(\mathrm{CDCl}_{3}\right) 152(\mathrm{t}, 3 \mathrm{H}, J 7.0), 4.60(\mathrm{q}, 2 \mathrm{H}, J$ 7.0), $7.76(\mathrm{~d}, 1 \mathrm{H}, J 2.1), 8.09(\mathrm{~d}, 1 \mathrm{H}, J 3.2)$ and $9.28(\mathrm{~s}, 1 \mathrm{H})$ (Found: C, 51.95; H, 3.8; N, 13.6. $\mathrm{C}_{9} \mathrm{H}_{8} \mathrm{~N}_{2} \mathrm{O}_{2} \mathrm{~S}$ requires: $\mathrm{C}$, $51.91 ; \mathrm{H}, 3.87$; N, $13.45 \%$ ).

5-Nitro-2,1,3-benzothiadiazole 3b. Thionyl chloride $(0.87$ $\mathrm{cm}^{3}, 12 \mathrm{mmol}$ ) was slowly added at $0{ }^{\circ} \mathrm{C}$ to a solution of 5 -nitro$o$-phenylenediamine $(1.53 \mathrm{~g}, 10 \mathrm{mmol})$ and triethylamine $(2.28$ $\left.\mathrm{cm}^{3}, 20 \mathrm{mmol}\right)$ in DMF $\left(15 \mathrm{~cm}^{3}\right)$. The resulting mixture was stirred for $3 \mathrm{~h}$ at room temperature after which it was diluted with water and extracted with chloroform $\left(2 \times 50 \mathrm{~cm}^{3}\right)$. The combined extracts were washed with aq. sodium hydrogen carbonate, water and brine, dried $\left(\mathrm{Na}_{2} \mathrm{SO}_{4}\right)$ and evaporated under reduced pressure. The residue was purified by silica gel column chromatography (chloroform-hexane) to give the title compound $3 \mathrm{~b}(0.80 \mathrm{~g}$, yield $44 \%)$, mp $133^{\circ} \mathrm{C} ; \delta_{\mathrm{H}}\left(\mathrm{CDCl}_{3}\right) 8.17$ $(\mathrm{d}, 1 \mathrm{H}, J 9.27), 8.44(\mathrm{dd}, 1 \mathrm{H}, J 9.58,2.20)$ and $8.99(\mathrm{~d}, 1 \mathrm{H}, J$ 1.95); $\delta_{\mathrm{C}}\left(\mathrm{CDCl}_{3}\right) 118.23(\mathrm{C}-7), 122.42$ (C-4), 122.86 (C-6), $148.46(\mathrm{C}-5), 153.26$ and $156.30 ; v_{\max }(\mathrm{KBr}) / \mathrm{cm}^{-1} 1556,1508$, $1344,1303,902,842,820$ and $736 ; \lambda_{\max }\left(\mathrm{CH}_{2} \mathrm{Cl}_{2}\right) / \mathrm{nm} 316$ and $265 ; m / z 183\left[\mathrm{M}^{+}\left({ }^{34} \mathrm{~S}\right), 9 \%\right], 182\left[\mathrm{M}^{+}\left({ }^{33} \mathrm{~S}\right), 5\right], 181\left[\mathrm{M}^{+}\right.$ $\left.\left({ }^{32} \mathrm{~S}\right), 100\right], 135\left(\mathrm{M}^{+}-\mathrm{NO}_{2}, 42\right)$ and $123(48)$ [Found (HRMS): M, 180.9941. Calc. for $\left.\mathrm{C}_{6} \mathrm{H}_{3} \mathrm{O}_{2} \mathrm{~S}: M, 180.9947\right]$.

\section{4-Nitro-2,1,3-benzoselenadiazole 5a}

Nitration of 2,1,3-benzoselenadiazole with $\mathrm{HNO}_{3}-\mathrm{H}_{2} \mathrm{SO}_{4}$ gave the nitro compound 5a $(89 \%), \mathrm{mp} 227^{\circ} \mathrm{C} ; \delta_{\mathrm{H}}\left(\left[^{2} \mathrm{H}_{6}\right]\right.$ acetone $)$ $7.82(\mathrm{dd}, 1 \mathrm{H}, J 9.00,7.17), 8.27(\mathrm{dd}, 1 \mathrm{H}, J 9.01,1.08)$ and $8.45\left(\mathrm{~d}, 1 \mathrm{H}, J\right.$ 7.02); $v_{\max }(\mathrm{KBr}) / \mathrm{cm}^{-1} 1526,1506,1432,1320$, 1292,814 and $734 ; \lambda_{\max }\left(\mathrm{CH}_{2} \mathrm{Cl}_{2}\right) / \mathrm{nm} \mathrm{340;m/z} 231\left[\mathrm{M}^{+}\left({ }^{82} \mathrm{Se}\right)\right.$, $21 \%], 230\left[\mathrm{M}^{+}\left({ }^{81} \mathrm{Se}\right), 10\right], 229\left[\mathrm{M}^{+}\left({ }^{80} \mathrm{Se}\right), 100\right], 228\left[\mathrm{M}^{+}\right.$ $\left.\left({ }^{79} \mathrm{Se}\right), 7\right], 227\left[\mathrm{M}^{+}\left({ }^{78} \mathrm{Se}\right), 50\right], 226\left[\mathrm{M}^{+}\left({ }^{77} \mathrm{Se}\right), 21\right], 225\left[\mathrm{M}^{+}\right.$ $\left.\left({ }^{76} \mathrm{Se}\right), 21\right], 192(42), 183\left(\mathrm{M}^{+}-\mathrm{NO}_{2}, 37\right), 171(38)$ and 156 (40) [Found (HRMS): M, 228.9405. Calc. for $\mathrm{C}_{6} \mathrm{H}_{3} \mathrm{~N}_{3} \mathrm{O}_{2} \mathrm{Se}$ : $M, 228.9391]$.

\section{5-Nitro-2,1,3-benzoselenadiazole $5 \mathrm{~b}$}

Acetic acid $\left(200 \mathrm{~cm}^{3}\right)$ was added to a stirred mixture of 5-nitro$o$-phenylenediamine $(7.65 \mathrm{~g}, 50 \mathrm{mmol})$ and selenium(Iv) oxide $(5.55 \mathrm{~g}, 50 \mathrm{mmol})$ in ethanol $\left(500 \mathrm{~cm}^{3}\right)$. The mixture was vigorously stirred at reflux temperature for $2 \mathrm{~h}$ after which it was evaporated under reduced pressure. The residue was dissolved in ethyl acetate $\left(200 \mathrm{~cm}^{3}\right)$ and the solution was washed with aqueous sodium hydrogen carbonate, water and brine, dried $\left(\mathrm{Na}_{2} \mathrm{SO}_{4}\right)$, filtered and evaporated under reduced pressure to give the crude product. This could be used in the next reaction without further purification (yield $70 \%$ ), mp $224{ }^{\circ} \mathrm{C} ; \delta_{\mathrm{H}}\left(\mathrm{CDCl}_{3}\right) 8.00(\mathrm{dd}, 1 \mathrm{H}, J 9.77,0.61), 8.30(\mathrm{dd}, 1 \mathrm{H}, J$ $9.76,2.14)$ and $8.84(\mathrm{dd}, 1 \mathrm{H}, J 2.44,0.61) ; v_{\max }(\mathrm{KBr}) / \mathrm{cm}^{-1} 1542$, $1500,1338,1272,840,760$ and $732 ; \lambda_{\max }\left(\mathrm{CH}_{2} \mathrm{Cl}_{2}\right) / \mathrm{nm} 316 ; \mathrm{m} / \mathrm{z}$ $231\left[\mathrm{M}^{+}\left({ }^{82} \mathrm{Se}\right), 19 \%\right], 230\left[\mathrm{M}^{+}\left({ }^{81} \mathrm{Se}\right), 8\right], 229\left[\mathrm{M}^{+}\left({ }^{80} \mathrm{Se}\right)\right.$, $100], 228\left[\mathrm{M}^{+}\left({ }^{79} \mathrm{Se}\right), 4\right], 227\left[\mathrm{M}^{+}\left({ }^{78} \mathrm{Se}\right), 48\right], 226\left[\mathrm{M}^{+}\left({ }^{77} \mathrm{Se}\right)\right.$, $18], 225\left[\mathrm{M}^{+}\left({ }^{76} \mathrm{Se}\right), 19\right], 192(42), 183\left(\mathrm{M}^{+}-\mathrm{NO}_{2}, 37\right), 171$ (18) and 156 (23); [Found (HRMS): M, 228.948. Calc. for $\mathrm{C}_{6} \mathrm{H}_{3} \mathrm{~N}_{3} \mathrm{O}_{2}$ Se: $\left.M, 228.9391\right]$.

The pyrroles $4 a, 6 a$ and the pyrimidine $N$-oxides $4 \mathbf{b}, 6 \mathbf{b}$ were prepared by a similar procedure to that described in the preparation of $2 \mathbf{a}$, under the conditions described in Table 2.

Ethyl pyrrolo[3,4-e][2,1,3]benzothiadiazole-6-carboxylate 4a. Yield $33 \%$; mp $183{ }^{\circ} \mathrm{C} ; \delta_{\mathrm{H}}\left(\mathrm{CDCl}_{3}\right) 1.49(\mathrm{t}, 3 \mathrm{H}, J 7.17), 4.43$ (q, $2 \mathrm{H}, J 7.12), 7.62(\mathrm{~d}, 1 \mathrm{H}, J 9.46), 7.96(\mathrm{~d}, 1 \mathrm{H}, J 3.36), 8.19$ $(\mathrm{d}, 1 \mathrm{H}, J 9.46)$ and $10.1(\mathrm{~s}, 1 \mathrm{H}) ; \delta_{\mathrm{C}}\left(\mathrm{CDCl}_{3}\right) 14.39\left(\mathrm{CH}_{2} \mathrm{CH}_{3}\right)$, $60.20\left(\mathrm{CH}_{2} \mathrm{CH}_{3}\right), 115.24,115.58,118.29$ (C-5), 119.14 (C-8), $125.00,125.48(\mathrm{C}-4), 150.39,153.92$ and $160.432(\mathrm{C}=\mathrm{O})$; $v_{\max }(\mathrm{KBr}) / \mathrm{cm}^{-1} 3212,1688,1398,1338,1274,1170$ and 1132 ; $\lambda_{\max }\left(\mathrm{CH}_{2} \mathrm{Cl}_{2}\right) / \mathrm{nm} \mathrm{358,308,} 295$ and $267 ; \mathrm{m} / \mathrm{z} 249\left[\mathrm{M}^{+}\left({ }^{34} \mathrm{~S}\right)\right.$, $6 \%], 248\left[\mathrm{M}^{+}\left({ }^{33} \mathrm{~S}\right), 14\right], 247\left[\mathrm{M}^{+}\left({ }^{32} \mathrm{~S}\right), 100\right]$ and 201 (Found: $\mathrm{C}, 53.7, \mathrm{H}, 3.7, \mathrm{~N}, 17.1 . \mathrm{C}_{11} \mathrm{H}_{9} \mathrm{~N}_{3} \mathrm{O}_{2} \mathrm{~S}$ requires $\mathrm{C}, 53.43 ; \mathrm{H}$, $3.67 ; \mathrm{N}, 16.99 \%$ ).
Ethyl pyrimido[5,4-e][2,1,3]benzothiadiazole-9-carboxylate 6-oxide 4b. Yield $21 \% ; \mathrm{mp} 181^{\circ} \mathrm{C} ; \delta_{\mathrm{H}}\left(\mathrm{CDCl}_{3}\right) 1.48(\mathrm{t}, 3 \mathrm{H}, J$ 7.08 ), 4.67 (q, $2 \mathrm{H}, J 7.16), 8.37$ (d, $1 \mathrm{H}, J 9.77), 8.77$ (d, $1 \mathrm{H}, J$ 9.77) and $9.31(\mathrm{~s}, 1 \mathrm{H}) ; \delta_{\mathrm{C}}\left(\mathrm{CDCl}_{3}\right) 13.98\left(\mathrm{CH}_{2} \mathrm{CH}_{3}\right), 63.13$ $\left(\mathrm{CH}_{2} \mathrm{CH}_{3}\right), 116.20,120.66$ (C-4), $128.48(\mathrm{C}-5), 142.80,145.99$, $146.24(\mathrm{C}-7), 148.91,154.46$ and $164.79(\mathrm{C}=\mathrm{O}) ; v_{\max }(\mathrm{KBr}) / \mathrm{cm}^{-1}$ $1746,1276,1244,1198,1184,1144,1014$ and 832; $\lambda_{\max }\left(\mathrm{CH}_{2} \mathrm{Cl}_{2}\right) / \mathrm{nm} \mathrm{405,} 290$ and $254 ; m / z 278\left[\mathrm{M}^{+}\left({ }^{34} \mathrm{~S}\right), 7 \%\right]$, $277\left[\mathrm{M}^{+}\left({ }^{33} \mathrm{~S}\right), 16\right], 276\left[\mathrm{M}^{+}\left({ }^{32} \mathrm{~S}\right), 100\right], 231\left(\mathrm{M}^{+}\right.$- OEt, 47), 204 (79) and 188 (62) [Found (HRMS): M, 276.0314. Calc. for $\mathrm{C}_{11} \mathrm{H}_{8} \mathrm{~N}_{4} \mathrm{O}_{3} \mathrm{~S}: M, 276.0318$ ] (Found: C, 47.85, H, 3.0, N, 20.3. $\mathrm{C}_{11} \mathrm{H}_{8} \mathrm{~N}_{4} \mathrm{O}_{3} \mathrm{~S}$ requires: $\mathrm{C}, 47.82 ; \mathrm{H}, 2.92 ; \mathrm{N}, 20.28 \%$ ).

Ethyl pyrrolo $[3,4-e][2,1,3]$ benzoselenadiazole-6-carboxylate 6a. Yield $56 \% ; \mathrm{mp} 248^{\circ} \mathrm{C} ; \delta_{\mathrm{H}}\left(\mathrm{CDCl}_{3}\right) 1.43(\mathrm{t}, 3 \mathrm{H}, J 7.17), 4.43$ (q, $2 \mathrm{H}, J 7.12$ ), 7.42 (d, $1 \mathrm{H}, J 9.77), 8.02(\mathrm{~d}, 1 \mathrm{H}, J 2.74), 8.13$ $(\mathrm{d}, 1 \mathrm{H}, J 9.77)$ and $12.1(\mathrm{~s}, 1 \mathrm{H}) ; v_{\max }(\mathrm{KBr}) / \mathrm{cm}^{-1} 2896,1682$, $1432,1322,1274,1178$ and 1142; $\lambda_{\max }\left(\mathrm{CH}_{2} \mathrm{Cl}_{2}\right) / \mathrm{nm} \mathrm{378,294}$, 288 and $264 ; m / z 297\left[\mathrm{M}^{+}\left({ }^{82} \mathrm{Se}\right), 22 \%\right], 296\left[\mathrm{M}^{+}\left({ }^{81} \mathrm{Se}\right), 14\right]$, $295\left[\mathrm{M}^{+}\left({ }^{80} \mathrm{Se}\right), 100\right], 294\left[\mathrm{M}^{+}\left({ }^{79} \mathrm{Se}\right), 7\right], 293\left[\mathrm{M}^{+}\left({ }^{78} \mathrm{Se}\right), 54\right]$, $292\left[\mathrm{M}^{+}\left({ }^{77} \mathrm{Se}\right), 20\right], 291\left[\mathrm{M}^{+}\left({ }^{76} \mathrm{Se}\right), 19\right]$ and $249\left(\mathrm{M}^{+}-\right.$ $\mathrm{EtOH}$, 88) [Found (HRMS): M, 294.9853. Calc. for $\mathrm{C}_{11} \mathrm{H}_{9} \mathrm{~N}_{3} \mathrm{O}_{2} \mathrm{Se}: M, 294.9861$ ] (Found: $\mathrm{C}, 44.2, \mathrm{H}, 3.0, \mathrm{~N}, 14.1$. $\mathrm{C}_{11} \mathrm{H}_{9} \mathrm{~N}_{3} \mathrm{O}_{2}$ Se requires $\mathrm{C}, 44.91 ; \mathrm{H}, 3.08 ; \mathrm{N}, 14.28 \%$ ).

Ethyl pyrimido[5,4-e][2,1,3] benzoselenadiazole-9-carboxy]ate 6-oxide $6 \mathrm{~b}$. Yield $28 \% ; \mathrm{mp} 248{ }^{\circ} \mathrm{C} ; \delta_{\mathrm{H}}\left(\mathrm{CDCl}_{3}\right) 1.47(\mathrm{t}$, $3 \mathrm{H}, J 7.08$ ), 4.64 (q, $2 \mathrm{H}, J 7.16), 8.20$ (d, $1 \mathrm{H}, J 9.77), 8.67$ (d, $1 \mathrm{H}, J 9.77)$ and $9.29(\mathrm{~s}, 1 \mathrm{H}) ; \delta_{\mathrm{C}}\left(\mathrm{CDCl}_{3}\right) 14.02\left(\mathrm{CH}_{2} \mathrm{CH}_{3}\right)$, $63.05\left(\mathrm{CH}_{2} \mathrm{CH}_{3}\right), 117.66,120.47$ (C-4), 130.65 (C-5), 142.31, $146.55,146.77(\mathrm{C}-7), 153.59,158.93$ and $165.24(\mathrm{C}=\mathrm{O})$; $v_{\max }(\mathrm{KBr}) / \mathrm{cm}^{-1} 1742,1278,1242,1196,1016,828$ and 770 ; $\lambda_{\max }\left(\mathrm{CH}_{2} \mathrm{Cl}_{2}\right) / \mathrm{nm} 419,319$ and $261 ; m / z 326\left[\mathrm{M}^{+}\left({ }^{82} \mathrm{Se}\right), 18 \%\right]$, $325\left[\mathrm{M}^{+}\left({ }^{81} \mathrm{Se}\right), 16\right], 324\left[\mathrm{M}^{+}\left({ }^{80} \mathrm{Se}\right), 100\right], 323\left[\mathrm{M}^{+}\left({ }^{79} \mathrm{Se}\right), 7\right]$, $322\left[\mathrm{M}^{+}\left({ }^{78} \mathrm{Se}\right), 49\right], 321\left[\mathrm{M}^{+}\left({ }^{77} \mathrm{Se}\right), 18\right], 320\left[\mathrm{M}^{+}\left({ }^{76} \mathrm{Se}\right), 20\right]$, $279\left(\mathrm{M}^{+}\right.$- OEt, 40), 252 (49) and 236 (15) [Found (HRMS): $\mathrm{M}$, 323.9759. Calc. for $\left.\mathrm{C}_{11} \mathrm{H}_{8} \mathrm{~N}_{4} \mathrm{O}_{3} \mathrm{Se}: M, 323.9762\right]$ (Found: $\mathrm{C}, 41.3, \mathrm{H}, 2.7, \mathrm{~N}, 17.1 . \mathrm{C}_{11} \mathrm{H}_{8} \mathrm{~N}_{4} \mathrm{O}_{3}$ Se requires $\mathrm{C}, 40.88 ; \mathrm{H}$, $2.50 ; \mathrm{N}, 17.34 \%$ ).

\section{Acknowledgements}

This work was partially supported by a Grant-in-Aid for Scientific Research from the Ministry of Education, Science, Sport and Culture, Japan.

\section{References}

1 For some leading references and recent advancements see: $(a)$ D. H. R. Barton and S. Z. Zard, J. Chem. Soc, Chem. Commun., 1985, 1098; (b) D. H. R. Barton, J. Kervagoret and S. Z. Zard, Tetrahedron, 1990, 46, 7587; (c) N. Ono, E. Muratani and T. Ogawa, J. Heterocycl. Chem., 1991, 28, 2053; (d) N. Ono, H. Katayama, S. Nishiyama and T. Ogawa, J. Heterocycl. Chem., 1994, 31, 707; (e) M. Hori and M. Mori, J. Org. Chem., 1995, 60, 1480; $(f)$ A. R. Katritzky, J. Li and M. F. Gordeev, Synthesis, 1994, 93; ( $g$ ) T. D. Lash, J. R. Bellettini, J. A. Bastian and K. B. Couch, Synthesis, 1994, 170; (h) A. J. G. Baxter, J. Fuher and S. J. Teague, Synthesis, 1994, 207; (i) P. La Porta, L. Capuzzi and F. Bettarini, Synthesis, 1994, 287; $(j)$ H. Frey, Synlett., 1994, 1007; $(k)$ B. P. Coppola, M. C. Noe, D. J. Schwartz, R. L. II Abdon and B. M. Trost, Tetrahedron, 1994, 50, 93; (l) G. Haake, D. Struve and F. P. Montforts, Tetrahedron Lett., 1994, 35, 9703; (m) J. T. Gupton, D. A Krolikowski, R. H. Yu, S. W. Riesinger and J. A. Sikorski, J. Org Chem., 1990, 55, 4735 .

2 (a) C. K. Sha, C. P. Tsou, Y. C. Li, R. S. Lee, F. Y. Tsai and R. H. Yeh, J. Chem. Soc., Chem. Commun., 1988, 1081; (b) F. Garcia and C. Galvez, Synthesis, 1985, 143.

3 (a) N. Ono, H. Hironaga, K. Simizu, K. Ono, K. Kuwano and T. Ogawa, J. Chem. Soc., Chem. Commun., 1994, 1019; (b) N. Ono, H. Kawamura, M. Bougauchi and K. Maruyama, J. Chem. Soc., Chem. Commun., 1989, 1580; (c) N. Ono, H. Hironaga, K. Ono, S. Kaneko, T. Murashima, T. Ueda, C. Tsukamura and T. Ogawa, J. Chem. Soc., Perkin Trans. 1, 1996, 417.

4 For a general survey of nucleophilic aromatic substitution see: (a) 
F. Terrier, Nucleophilic Aromatic Displacement, VCH Publishers, Inc., New York, 1991; (b) M. MaKosza, Synthesis, 1991, 103.

5 N. Ono, H. Tomita and K. Maruyama, J. Chem. Soc., Perkin Trans. 2, 1992, 2453; (b) T. D. Lash and B. H. Novak, Angew. Chem., Int. Ed. Engl., 1995, 34, 683 .

6 H. Uno, T. Kinoshita, K. Matsumoto, T. Murashima, T. Ogawa and N. Ono, J. Chem. Res., 1995, 76.

7 D. J. Brown, Comprehensive Heterocyclic Chemistry, eds. A. R. Katritzky and C. W. Rees, Pergamon, Oxford, 1984, vol. 3, pp. 106140.

8 M. Ogata, H. Watanabe, K. Tori and H. Kano, Tetrahedron Lett., $1964,19$.

9 W. B. Cowden and N. W. Jacobsen, Aust. J. Chem., 1979, 32, 2049.
10 (a) S. Shi, T. J. Katz, B. V. Yang and L. Liu, J. Org. Chem., 1995, 60, 1285; (b) L. M. Weinstock and I. Shinkai, Comprehensive Heterocyclic Chemistry, eds. A. R. Katritzky and C. W. Rees, Pergamon, Oxford, 1984, vol 6, p 537; (c) K. Pilgram, J. Org. Chem., $1970,35,1165$

11 U. Schöllkopf, D. Hoppe and R. Jentsch, Chem. Ber., 1975, 108, 1580

Paper 5/07760E

Received 28th November 1995 Accepted 5th February 1996 